\title{
Mathematical Analysis of a Model for Human Immunodeficiency Virus (HIV) Endemicity
}

\author{
JAMES, A; EGUDA, FY \\ Department of Mathematics, Federal University Dutse, Jigawa State \\ Email: jamesandy7772@gmail.com
}

\begin{abstract}
The objective of this paper is to present a mathematical model formulated to investigate the dynamics of human immunodeficiency virus (HIV). The disease free equilibrium of the model was found to be locally and globally asymptotically stable. The endemic equilibrium point exists and it was discovered that the endemic equilibrium point is globally asymptotically stable; suggestion was also made in the research in regards to protection during sexual intercourse especially in a sexually active population.
\end{abstract}

DOI: https://dx.doi.org/10.4314/jasem.v21i7.23

Copyright @ 2017 Ibrahim et al. This is an open access article distributed under the Creative Commons Attribution Non-Commercial License (CC-BY-NC), which permits unrestricted use, distribution, and reproduction in any medium, provided the original work is properly cited.

Received 02 September 2017, received in revised form 19 October 2017, accepted 22 November 2017.

Keywords: Analysis, Equilibrium, Stability, Endemicity, Mathematical Model

HIV is a virus that spread through body fluids (which majorly involves the exchange of body fluids during sexual intercourse) that affects specific cells of the immune system called CD4 cells or T cells. With time, so many of these cells are destroyed and the infection leads to Acquired Immunodeficiency Syndrome (AIDS) (Weiss, 1993). AIDS can be said to be a condition in humans in which progressive failure of the immune system allows for life threatening opportunistic infections and cancers to thrive (Weiss, 1993). Without treatment, average survival time after infection with HIV is estimated to be 9 to 11 years depending on the HIV subtype and it usually overwhelms the immune system (UNAIDS, WHO 2007).

The stages of progression of HIV include; Acute infection which last within two to four weeks after infection. During this period, one may feel flu-like symptoms called acute retroviral syndrome (ARS) or primary HIV infection (CDC, 2015). One has the highest likelihood to spread HIV at this stage due to the amount of viral load in the blood. After this period, the immune system begins to build up again but it may not return to pre infection levels (CDC, 2015). Clinical latency (inactivity or dormancy) sometimes called the asymptomatic HIV infection. At this stage, the viruses reproduce but at very low levels, there may be no symptoms which can last up to a decade. During this phase of infection, HIV is active within the lymph nodes (Burton et al, 2002). However, with anti-retroviral therapy (ART), latency can last for several decades and also reduces the tendency to transmit the infection. As time progresses, the viral load begins to increase and the CD4 cell count begins to drop. And symptoms may begin to appear (CDC, 2015). Acquired Immunodeficiency Syndrome (AIDS). This is the final stage. The immune system is completely damaged with the CD4 cells below 200 cells per $1 \mathrm{~mm}^{3}$ of blood, one becomes vulnerable to infections and infection related cancers called opportunistic illnesses with life expectancy of about 3years. When one is hit by a dangerous opportunistic illness, which is mainly determined by the prevalent infection in the geographical area of the patient, life expectancy drops to a year (CDC, 2015). HIV has no cure or vaccination. However treatment is available. Highly active retroviral therapy has been resoundingly successful at reducing morbidity and mortality of infected individuals (May and Ingle, 2011). Anderson et al (1986), May and Anderson (1987) analysed a simple deterministic HIV model, without treatment, using a bilinear incidence function. The population was divided into three classes; the susceptibles (S), the infected (I) and the AIDS patients, (A). They took the assumption that only those in the I class can spread the disease. The system below was used to describe the dynamics of the disease. Naresh et al (2008) formulated a model with a population of varying size and immigration of infectives. Despite the introduction of different control strategies including anti-retroviral therapy (ART), HIV remains a global health challenge in the world. In this paper, a mathematical model is formulated and analysed to investigate the transmission pattern of HIV in a sexually active population in order to understand and 
reduce the threat posed by the disease. Also, global properties will be discussed.

\section{MATERIALS AND METHODS}

The total sexually active population at time $t$ is denoted by $N(t)$ and it is divided into 2 mutually exclusive compartments. Let $S(t)$ and $H_{1}(t)$ be the two (2) compartments which represent the populations of susceptible individuals and individuals with human immunodeficiency virus (HIV). So that $N(t)=S(t)+H_{1}(t)$.

The susceptible population $\mathrm{S}(\mathrm{t})$ is generated by the recruitment of individuals (assumed susceptible) into the population at a rate $\Lambda$. Infectious interactions in the population are modeled using a standard incidence function typically written in the form $\frac{\beta I}{N}$ where the effective contact rate $\beta$ increases linearly with the population size N. Susceptible individuals acquire HIV infection following effective contacts with people infected with HIV that is those in the $\mathrm{H}_{1}(\mathrm{t})$ class at a rate given by $\lambda_{H}=\beta_{H} \frac{H_{1}}{N}$
Where $\beta_{H}$ is the effective contact rate for the transmission.

Mathematical Model Formulation: The population of individuals in the both classes has a mortality rate $\mu$ and those in $H_{1}$ classes has a disease induced death rate $d_{H 1}$. Combining all the afore-mentioned assumptions and definitions, the model for the transmission of HIV in a sexually active population is given by the following system of differential equations (1)

$\dot{S}=\Lambda-\lambda_{H} S-\mu S$

$\dot{H}_{1}=\lambda_{H} S-\left(\mu+d_{H 1}\right) H_{1}$

$\lambda_{H}$ is defined as $\lambda_{H}=\frac{\beta_{H} H_{1}}{N}$ and $N=S+H_{I}$

\section{Data Acquisition and Analysis}

Consider the region $D_{1}=\left\{\left(S, H_{1}\right) \in \mathbb{R}_{+}^{2}: N \leq \frac{\Lambda}{\mu}\right\}$. It can be shown that the set $D_{1}$ is positively invariant and a global attractor of all positive solution of the system (1).

Lemma 1 The region $D_{1}$ is positively invariant for the system (1)

Proof: The rate of change of the total population is given as

$\dot{N}=\dot{S}+\dot{H}_{1}=\Lambda-\mu\left(S+H_{1}\right)-d_{H 1} H_{1}$

$\dot{N}=\Lambda-\mu N-d_{H 1} H_{1}$

By standard comparison theorem, $\dot{N} \leq \Lambda-\mu N$

So we have $\dot{N}+\mu N \leq \Lambda$. Using the integrating factor method $N=N_{0} e^{-\mu t}+\frac{\Lambda}{\mu}\left[1-e^{-\mu t}\right]$

If $\mathrm{N}_{0} \leq \frac{\Lambda}{\mu}$ then $\mathrm{N} \leq \frac{\Lambda}{\mu}$ so, $\mathrm{D}_{1}$ is a positively invariant set under the flow described in (1). Hence, no solution path leaves through and boundary of $D_{1}$. Also, since solution paths cannot leave $D_{1}$, solutions remain non-negative for non-negative initial conditions. Solutions exist for all time t. In this region, the model (1) is said to be well posed mathematically and epidemiologically.

Lemma 2 Let the initial data for the model (1) be $S(0)>0, H_{l}(0)>0$, then the solution $S(t)$, $H_{l}(t)$ with positive initial data will remain positive for all time $t>0$

Proof: Let $t_{1}=\sup \left\{t>0: S(t)>0, H_{1}(t)>0\right\}>0, \dot{S}=\Lambda-\lambda_{H} S-\mu S=\Lambda-\left(\lambda_{H}+\mu\right) S$

To solve the ODE using the integrating factor method

$$
\begin{aligned}
& I . F=\exp \left[\mu t+\left\{\int_{0}^{t} \lambda_{H}(\tau) d(\tau)\right\}\right] \\
& \frac{d}{d t}\left[S(t) \exp \left\{\mu t+\int_{0}^{t} \lambda_{H}(\tau) d(\tau)\right\}\right]=\Lambda\left[\exp \left\{\mu t+\int_{0}^{t} \lambda_{H}(\tau) d(\tau)\right\}\right] \\
& S\left(t_{1}\right) \exp \left\{\mu t_{1}+\int_{0}^{t_{1}} \lambda_{H}(\tau) d(\tau)\right\}=S(0)+\int_{0}^{t_{1}} \Lambda\left[\exp \left\{\mu y+\int_{0}^{y} \lambda_{H}(\tau) d(\tau)\right\}\right] d y \\
& S\left(t_{1}\right)=S(0) \exp \left\{-\mu t_{1}-\int_{0}^{t_{1}} \lambda_{H}(\tau) d(\tau)\right\} \\
& +\left[\exp \left\{-\mu t_{1}-\int_{0}^{t_{1}} \lambda_{H}(\tau) d(\tau)\right\}\right] \int_{0}^{t_{1}} \Lambda\left[\exp \left\{\mu y+\int_{0}^{y} \lambda_{H}(\tau) d(\tau)\right\}\right] d y>0
\end{aligned}
$$

For $\dot{H}_{1}=\lambda_{H} S-\left(\mu+d_{H 1}\right) H_{1}$ we have that $\dot{H}_{1} \geq-\left(\mu+d_{H 1}\right) H_{1}$ 
Similarly, we can show that $\mathrm{H}_{1}(\mathrm{t})>0$

Local Stability of Disease-free Equilibrium (DFE): The model 1 has a disease-free equilibrium obtained by setting the right hand side of the model to zero given by

$\xi_{(0, H)}=\left(S^{*}, H_{1}^{*}\right)=\left(\frac{\Lambda}{\mu}, 0\right)$

The stability of $\xi_{0}$ is established using the next generation operator method on the system (1). Using the notation in Van den Driessche and Watmough (2002), Andrawus and Eguda(2017) the matrices F and $\mathrm{V}$ for the new infection terms and the remaining transfer terms respectively, are respectively given as $F=\left(\beta_{H}\right), V=\left(\mu+d_{H 1}\right)$

The spectral radius given by $\rho\left(F V^{-1}\right)=\frac{\beta_{H}}{\mu+d_{H 1}}=R_{H}$

The next result follows from theorem 2 of Van den Driessche and Watmough (2002).

Lemma 3 The DFE of model $(1), \xi_{(0, H)}$ is locally asymptotically stable if $R_{H}<1$ and unstable if $R_{H}>1$ The threshold quantity $R_{H}$ is the basic reproduction number for the HIV. Speaking biologically, Lemma (3) implies that HIV can be eliminated from the population when $\left(R_{H}<1\right)$ if the initial sizes of the sub-population of the sub-model are in the region of attraction of $\xi_{(0, H)}$.
It was also established from Lemma (3) that if the DFE actually exists, it is locally asymptotically stable if and only if $\mathrm{R}_{\mathrm{H}}<1$.

Existence and Local Stability of Endemic Equilibrium Point (EEP) of the Model: Let the EEP of model (1) be denoted by $\xi_{(1, H)}=\left(S^{* *}, H_{1}^{* *}\right)$. The equations in 1 are solved in terms of the force of infection at steady state and they are given as $S^{* *}=\frac{\Lambda}{\lambda_{H}^{* *}+\mu}$ and $H_{1}^{* *}=$ $\frac{\lambda_{H}^{* *} S^{* *}}{\mu+d_{H 1}}$. So we have

$$
\begin{aligned}
& N^{* *}=S^{* *}+H_{1}^{* *} \text { which gives } N^{* *} \\
& =\frac{\Lambda\left(\mu+d_{H 1}+\lambda_{H}^{* *}\right)}{\left(\mu+d_{H 1}\right)\left(\mu+\lambda_{H}^{* *}\right)}
\end{aligned}
$$

Now $\lambda_{H}^{* *}=\frac{\beta_{H} H_{1}^{* *}}{N^{* *}}=\beta_{H}-\mu-d_{H 1}$ after substituting $H_{1}^{* *}$ and $N^{* *}$

Therefore we have $\lambda_{H}^{* *}+\left(\mu+d_{H 1}\right)\left[1-R_{H}\right]=0$

If $\mathrm{R}_{\mathrm{H}}>1$, then $\left(\mu+\mathrm{d}_{\mathrm{H} 1}\right)\left[1-\mathrm{R}_{\mathrm{H}}\right]<0$. So, the system (1) has a unique endemic equilibrium if

$\mathrm{R}_{\mathrm{H}}>1$. Also, for the case where $\mathrm{d}_{\mathrm{H} 1}=0$ and $N=\frac{\Lambda}{\mu}$, we have $\lambda_{H}^{* *}+\mu\left(1-R_{H}\right)$

We also have that a unique EEP exists for $\mathrm{R}_{\mathrm{H}}>1$; and if $\mathrm{R}_{\mathrm{H}}<1$, then only the DFE exists.

\section{Local Asymptotic Stability of EEP}

Jacobian of the system (1) evaluated at the EEP is given as $J\left(\xi_{(1, H)}\right)=\left(\begin{array}{cc}-\frac{\beta_{H} H_{1}^{* 2}}{N^{* *}{ }^{2}}-\mu & -\frac{\beta_{H} s^{* *}{ }^{2}}{N^{* *}{ }^{2}} \\ \frac{\beta_{H} H_{1}^{* 2}}{N^{* * 2}} & \frac{\beta_{H} S^{* 2^{2}}}{N^{* * 2}}-\mu-d_{H 1}\end{array}\right)$

The determinant of $J\left(\xi_{(1, H)}\right)$ is given as Det $=\frac{\mu \beta_{H} H_{1}^{* *^{2}}}{N^{* *^{2}}}+\frac{d_{H 1} \beta_{H} H_{1}^{* * 2}}{N^{* *^{2}}}-\frac{\mu \beta_{H} S^{* * 2}}{N^{* * 2}}+\mu^{2}+\mu d_{H 1}$

Det> 0 if $\mathrm{R}_{\mathrm{H}}>1$

$$
\text { Det }=\frac{\beta_{H} H_{1}^{* *^{2}}}{N^{* *^{2}}}\left[\mu+d_{H 1}\right]+\mu^{2}+\mu d_{H 1}-\frac{\mu \beta_{H} S^{* *^{2}}}{N^{* *^{2}}}
$$

Using $S^{* *}=\frac{N^{* *}}{R_{H}}$ we have that $\operatorname{Det}=\frac{\beta_{H} H_{1}^{*{ }^{2}}}{N^{* *^{2}}}\left[\mu+d_{H 1}\right]+\mu \frac{\beta_{H}}{R_{H}}\left[1-\frac{1}{R_{H}}\right]$

$$
\begin{gathered}
\text { Trace is given as follows } \operatorname{Tr}=-\frac{\beta_{H} H_{1}^{* *}}{N^{* 2}}-\mu+\frac{\beta_{H} S^{* * 2}}{N^{* * 2}}-\mu-d_{H 1} \\
\text { With } N^{* *}=S^{* *}+H_{1}^{* *}, R_{H}=\frac{\beta_{H}}{\mu+d_{H 1}} \text { and } S^{* *}=\frac{N^{* *}}{R_{H}} \text {. We have } \operatorname{Tr}=-\mu-\frac{\beta_{H}}{R_{H}}\left[R_{H}-1\right]
\end{gathered}
$$

Trace $<0$ if $R_{H}>1$ since all the parameters are positive. So we have that the EEP is locally asymptotically stable if $\mathrm{R}_{\mathrm{H}}>1$

Global Stability of the DFE and EEP

Global Stability of DFE: We can use the Lyapunov function approach to provide a sufficient condition for the global stability of the DFE when $R_{H} \leq 1$. considering the model (1), we can claim the following

Theorem 1: The DFE of the model (1) is globally asymptotically stable (GAS) in $D_{1}$ whenever $R_{H}<1$

Proof: Consider the Lyapunov function $V=H_{1}$

Clearly, $V>0$ except at the DFE. Differentiating $V$ with respect to time, we have 
$\dot{V}=\dot{H}_{1}=H_{1}\left(\frac{\beta_{H} S}{N}-\left(\mu+d_{H 1}\right)\right)$. On $D_{1}, S \leq N \leq \frac{\Lambda}{\mu}$ hence, $\frac{S}{N} \leq 1$. So we have

$\dot{V} \leq H_{1}\left(\mu+d_{H 1}\right)\left(R_{H}-1\right)$

with the equality only at the DFE. For $R_{H} \leq 1$, we have that $\dot{V} \leq 0$ with that equality sign only limit set of each solution is contained in the largest invariant set for which $H_{1}=0$, which is the singleton DFE.

\section{Global Stability of EEP}

Theorem 2: The unique endemic equilibrium of the model (1) is GAS in $D_{I}$ whenever $R_{H}>1$

Proof: Consider the non-linear Lyapunov function (of the Goh-Volterra type)

$$
\begin{gathered}
F=S-S^{* *}-S^{* *} \ln \frac{S}{S^{* *}}+H_{1}-H_{1}{ }^{* *}-H_{1}^{* *} \ln \frac{H_{1}}{H_{1}{ }^{* *}} \dot{F}=\dot{S}-\frac{S^{* *}}{S} \dot{S}+\dot{H}_{1}-\frac{H_{1}^{* *}}{H_{1}} \dot{H}_{1} \\
\text { With } N=\frac{\Lambda}{\mu}, \lambda_{H}=\beta_{H} \frac{H_{1}}{N} \text { becomes } \tilde{\lambda}_{H}=\beta_{H} \frac{\Lambda}{\mu} H_{1}=\tilde{\beta}_{H} H_{1} \text { where } \tilde{\beta}_{H}=\beta_{H} \frac{\Lambda}{\mu} \text { we have }
\end{gathered}
$$

It can be shown from (1) that at steady state, $\Lambda=\tilde{\beta}_{H} H_{1}^{* *} S^{* *}+\mu S^{* *}$ and $\mu+d_{H 1}=\tilde{\beta}_{H} S^{* *}$

After some rigorous arithmetic we have

$$
\dot{F}=\mu S^{* *}\left(2-\frac{S^{* *}}{S}-\frac{S}{S^{* *}}\right)+\tilde{\beta}_{H} H_{1}^{* *} S^{* *}\left(2-\frac{S^{* *}}{S}-\frac{S}{S^{* *}}\right)
$$

Since arithmetic mean exceeds geometric mean, the following inequality

$$
2-\frac{s^{* *}}{S}-\frac{S}{S^{* *}} \leq 0 \text { will hold. }
$$

Thus, we have that $\dot{F} \leq 0$ for $R_{H}>1$. Hence; $F$ is a Lyapunov function in $D_{1}$

\section{RESULTS AND DISCUSSION}

A mathematical model for the transmission dynamics of Human Immunodeficiency Virus (HIV) in a human population is designed and used to assess the impact of control strategies on the cases. The Disease free equilibrium and Endemic equilibrium point of the model (1) was seen to be both locally and globally asymptotically stable.

Conclusions: Almost all population in the world is concerned about the rising prevalence of HIV which causes a lot of deaths in different communities. It still remains that there is no cure, neither a vaccine to control this epidemic. Almost all countries have increasingly recognized the need to find effective prevention and control strategies for the diseases and this is especially true in developing countries where treatment of HIV is almost always not available. This research work suggests the use of protection during sexual intercourse especially in active sexual population which will help reduce the number of cases.

\section{REFERENCES}

Anderson, RM; Medley, GF; May, RM; Johnson, AM (1986). A preliminary study of the transmission dynamics of HIV the causative agent of AIDS. IMA, J. Math. Appl. Med. Biol. 3:229-263.

Anderson, RM; May, RM (1987). Transmission Dynamics of HIV infection. Nature 326:137142 .
Andrawus, J; Eguda, FY (2017). Analysis of a Mathematical Model to Investigate the Dynamics of Dengue Fever J. Appl. Sci. Environ. Manage. June 2017 Vol. 21 (4) 626638

Burton, GF; Keele, BF., et al, (2002). Follicular dendritic cell contributions to HIV pathogenesis. Semin .Immuol. 14(4):275-284.

Centre for Disease Control (2015). www.cdc.gov/hiv/basics/whatishiv.html.

May, MT; Ingle, SM (2011). Life expectancy of HIV-positive adults: A review. Sexual health 8(4):526-533.

Van den Driessche, P; Watmough, J (2002). Reproduction numbers and sub-threshold endemic equilibria for compartmental models of disease transmission. Math.Biosci, 180:29-48.

Weiss, RA (1993). How does HIV cause AIDS? Science 260(5112):1273-1279.

World Health Organization. (2007) Global Incidence and Prevalence of Selected curable Sexually Transmitted Infections.

World Health Organisation (2015) https://www.aids.gov/hiv.aids.basics/hiv-aids101 Global statistics 2015 